\title{
4,7-二苯基-1,10-菲啰啉的合成研究
}

\author{
张红梅曹湖军张志勇彭娟娟崔宏宇 \\ 张天静雷钢铁* \\ (湘潭大学化学学院 环境友好化学与应用省部共建教育部重点实验室 湘潭 411105)
}

\begin{abstract}
摘要 以 4-苯基- 8 -硝基喹啉为起始原料, 经还原得到 4-苯基- 8 -氨基喹啉, 再以 $\mathrm{I}_{2} / \mathrm{KI}$ 为氧化剂, 在乙酸和盐酸的存在 下, 用 Skraup 法合成了 4,7-二苯基-1,10-菲啰啉. 化合物结构经 IR 和 ${ }^{1} \mathrm{H}$ NMR 得到了证实. 实验研究得到了最佳的合 成条件为: $n$ (3-氯苯丙酮) $: n$ (4-苯基- 8 -氨基喹啉 $)=1.5: 1, \mathrm{I}_{2} / \mathrm{KI}$ 用量为 $8 \%$, 反应温度 $120{ }^{\circ} \mathrm{C}$, 反应时间 $2.5 \mathrm{~h}$. 产品收 率可达 $82 \%$.
\end{abstract}

关键词 Skraup 反应; 4,7-二苯基-1,10-菲啰啉; 4-苯基-8-氨基喹啉

\section{Synthesis of 4,7-Diphenyl-1,10-phenanthroline}

\author{
Zhang, Hongmei \\ Cao, Hujun \\ Zhang, Zhiyong \\ Peng, Juanjuan \\ Cui, Hongyu
}

Zhang, Tianjing

Lei, Gangtie*

(Key Laboratory of Environmentally Friendly Chemistry and Applications of Ministry of Education, College of Chemistry, Xiangtan University, Xiangtan 411105)

\begin{abstract}
Diphenyl-1,10-phenanthroline was synthesized using 4-phenyl-8-nitroquinoline as raw material, which was reduced to 4-phenyl-8-aminoquinoline and then using $\mathrm{I}_{2} / \mathrm{KI}$ as oxidants in the presence of acetic acid and hydrochloric acid, by Skraup reaction. The structure of the product is confirmed by IR and ${ }^{1} \mathrm{H}$ NMR spectra. The results showed that the optimal conditions was $n$ (3-chloropropiophenone) $: n(4$-phenyl-8-aminoquinoline $)=1.5: 1, \mathrm{I}_{2} / \mathrm{KI}=8 \%$, reaction temperature $=120$ ${ }^{\circ} \mathrm{C}$, and reaction time $=2.5 \mathrm{~h}$. The yield of 4,7 -diphenyl-1,10-phenanthroline reached $82 \%$.
\end{abstract}

Keywords Skraup reaction; 4,7-diphenyl-1,10-phenanthroline; 4-phenyl-8-aminoquinoline

1,10 - 菲啰啉是研究最早和应用最广的含氮杂环鳌 合剂之一. 作为一个重要的配体, 其许多络合物在很多 领域内发挥了重要的作用 ${ }^{[1]}$. 例如, 以 1,10 -菲啰啉衍生 物为配体的络合物具有良好的光学性质, 可作为光敏剂 和光催化剂 ${ }^{[2]}$. 特别是对称二取代的 1,10-菲啰啉衍生 物, 由于此类物质能够保持配位体的两部分对称, 能很 好地避免与金属络合时产生立体异构现象, 因此是一类 特别重要而且值得研究的化合物. 本文用 Skraup 法合 成了 4,7-二苯基-1,10-菲啰啉, 重点考察了不同条件下 4,7-二苯基-1,10-菲啰啉的合成工艺.

先前的文献报道 ${ }^{[3-5]}$ 都是以砷酸作为氧化剂在浓硫 酸或浓盐酸中反应制得, 反应温度高达 $140{ }^{\circ} \mathrm{C}$, 反应很 剧烈, 能耗过大, 产率很低, 而且砷酸是一种剧毒物质, 严重污染环境, 因此需要探讨出一种环境友好且适宜操
作的氧化剂. 本文用乙酸代替了浓硫酸或浓磷酸, 采用 无毒的 $\mathrm{I}_{2} / \mathrm{KI}$ 代替砷酸作为氧化剂, 合成了 4,7 -二苯基1,10-菲啰啉，探讨了反应温度、反应时间等因素对其合 成的影响, 并寻找出最优化的合成条件. 合成路线如图 1 .

\section{1 实验部分}

\section{1 原料}

3-氯苯丙酮、碘、碘化钾、乙酸、盐酸均为分析纯, 4-苯基-8-硝基喹啉参考文献[3]自制.

\section{2 仪器}

Spectra One 型傅立叶变换红外光谱仪(美国 PE 公 司); Bruker-AV400 核磁共振仪(测试条件为室温 $25{ }^{\circ} \mathrm{C}$,

\footnotetext{
*E-mail: 1gt@xtu.edu.cn

Received July 6 2011; revised September 20 2011; aceepted November 15, 2011.

Project supported by the Science and Technology Planning Project of Hunan Province (No. 2008FJ3097)

湖南省科技计划项目(No. 2008FJ3097)资助项目.
} 


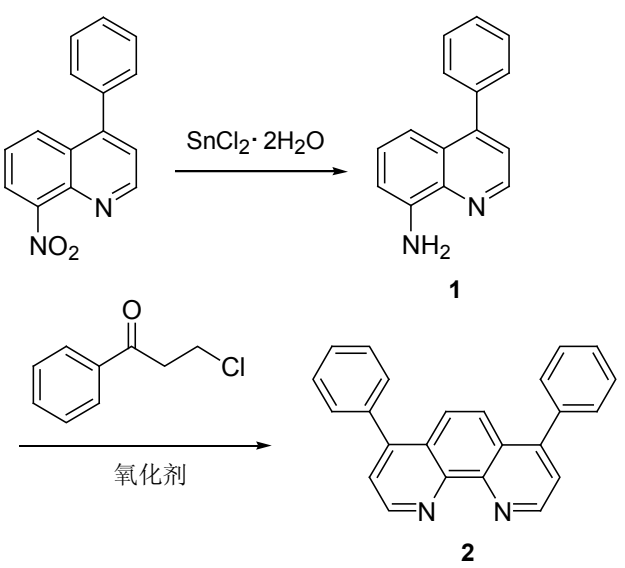

图 1 4,7-二苯基-1,10-菲啰啉的合成路线

Figure 1 The synthetic route of 4,7-diphenyl-1,10-phenanthroline

瑞士 Bruker 公司)

\section{3 合成方法}

\subsubsection{4-苯基-8-氨基喹啉(1)的合成}

在装有机械搅拌和冷凝管的 $500 \mathrm{~mL}$ 的四口瓶中, 加入 $34 \mathrm{~g}(0.15 \mathrm{~mol}) \mathrm{SnCl}_{2} \cdot 2 \mathrm{H}_{2} \mathrm{O}, 20 \mathrm{~mL}$ 浓磷酸, $20 \mathrm{~mL}$ 乙酸, $55{ }^{\circ} \mathrm{C}$ 搅拌 $10 \mathrm{~min}$, 再滴加 $12.5 \mathrm{~g}$ (0.05 mol)溶于乙 醇中的 4-苯基-8-硝基喹啉. $\mathrm{N}_{2}$ 保护, 搅拌升温至 $85{ }^{\circ} \mathrm{C}$ 反应 $3 \mathrm{~h}, 115{ }^{\circ} \mathrm{C}$ 反应 $2 \mathrm{~h}$. 冷却至室温, 搅拌下滴加稀氢 氧化钠溶液直至 $\mathrm{pH}$ 值为 $7 \sim 8$, 乙酸乙酯萃取, 无水硫 酸钠干燥过夜, 苯和石油醚重结晶, 得到纯化合物 $\mathbf{1}$ $10.8 \mathrm{~g}$, 淡黄绿色固体, 产率为 86.4\%. m.p. 88 90 ${ }^{\circ} \mathrm{C}$ (dec.)(文献值 ${ }^{[3]}$ : $\left.90 \sim 91{ }^{\circ} \mathrm{C}\right) ;{ }^{1} \mathrm{H}$ NMR $\left(\mathrm{CDCl}_{3}, 400 \mathrm{MHz}\right)$ $\delta: 7.38 \sim 7.29(\mathrm{~m}, 5 \mathrm{H}), 8.82(\mathrm{~d}, J=4.29 \mathrm{~Hz}, 1 \mathrm{H}, \mathrm{CH}), 7.54$ (d, $J=4.95 \mathrm{~Hz}, 1 \mathrm{H}, \mathrm{CH}), 7.24$ (d, $J=8.12 \mathrm{~Hz}, 1 \mathrm{H}, \mathrm{CH})$, 7.29 (d, $J=4.19 \mathrm{~Hz}, 1 \mathrm{H}, \mathrm{CH}), 6.9$ (d, $J=6.83 \mathrm{~Hz}, 1 \mathrm{H}$, $\mathrm{CH}), 5.14(\mathrm{~d}, J=12 \mathrm{~Hz}, 2 \mathrm{H}, 2 \times \mathrm{NH})$; IR (KBr) v: 3386, $3305,3040,1628,1511,1403,1353,1214,757,700 \mathrm{~cm}^{-1}$.

\subsubsection{4,7-二苯基-1,10-菲啰啉(2)的合成}

在装有机械搅拌和冷凝管的 $500 \mathrm{~mL}$ 的四口瓶中加 入 $22 \mathrm{~g}(0.1 \mathrm{~mol})$ 4-苯基- 8 -氨基喹啉, $100 \mathrm{~mL}$ 乙酸, 40 $\mathrm{mL}$ 盐酸. 把温度升到 $100{ }^{\circ} \mathrm{C}$, 然后缓慢(约 $30 \mathrm{~min}$ )加入 $10 \%$ (与原料的物质的量比)的氧化剂. 恒温摚拌 $30 \mathrm{~min}$ 后再缓慢加入 $22 \mathrm{~g}(0.13 \mathrm{~mol}) 3$-氯苯丙䣱, 加完后, 升 温至 $110{ }^{\circ} \mathrm{C}$, 继续反应 $2 \mathrm{~h}$. 冷却到室温, 倒入加有适量 冰块的烧杯中, 加氢氧化钠调制 $\mathrm{pH}=5 \sim 6$, 然后抽滤, 水洗至中性, 将固体置于 $60{ }^{\circ} \mathrm{C}$ 下干燥 $6 \mathrm{~h}$. 过柱提纯, 得到纯化合物 $223.2 \mathrm{~g}$, 浅黄色固体, 产率为 $69.9 \%$. m.p. $212 \sim 213{ }^{\circ} \mathrm{C}$ (dec.)(文献值 ${ }^{[3]}$ : $215 \sim 216{ }^{\circ} \mathrm{C}$ ); ${ }^{1} \mathrm{H}$ NMR $\left(\mathrm{CDCl}_{3}, 400 \mathrm{MHz}\right) \delta: 9.10(\mathrm{~d}, J=4 \mathrm{~Hz}, 2 \mathrm{H}, 2 \mathrm{CH})$, $8.13(\mathrm{~d}, J=8.4 \mathrm{~Hz}, 2 \mathrm{H}, 2 \mathrm{CH}), 8.03$ (d, $J=7.28 \mathrm{~Hz}, 4 \mathrm{H}$,
4CH), $7.58(\mathrm{t}, J=6.18 \mathrm{~Hz}, 4 \mathrm{H}, 4 \mathrm{CH}), 7.49(\mathrm{t}, J=3.6 \mathrm{~Hz}$, $4 \mathrm{H}, 4 \mathrm{CH})$; IR (KBr) v: 3043, 1563, 1532, 1488, 1445, $1366 \mathrm{~cm}^{-1}$.

\section{2 结果与讨论}

在此 Skraup 反应中，影响反应收率的因素主要有 反应温度、反应时间、3-氯苯丙酩和 4-苯基-8-氨基喹啉 进料物质的量比 $(r)$ 及催化剂的用量 $(m)$ (与 4-苯基-8-氨 基喹啉的物质的量比), 经单因素预实验分析, 确定了 正交实验的因素和水平见表 1 , 正交实验结果与分析见 表 2 .

表 1 正交实验的因素水平表

Table 1 The factor level of orthogonal experiment

\begin{tabular}{ccccc}
\hline \multirow{2}{*}{ Level } & \multicolumn{5}{c}{ Factor } \\
\cline { 2 - 5 } & $T /{ }^{\circ} \mathrm{C}$ & $t / \mathrm{h}$ & $r$ & $m / \%$ \\
\hline 1 & 100 & 1.5 & $1.25: 1$ & 4 \\
2 & 110 & 2.0 & $1.50: 1$ & 6 \\
3 & 120 & 2.5 & $1.75: 1$ & 8 \\
4 & 130 & 3.0 & $2.00: 1$ & 10 \\
\hline
\end{tabular}

表 2 正交实验结果及分析表

Table 2 The result and analysis of orthogonal experiment

\begin{tabular}{|c|c|c|c|c|c|}
\hline \multirow{2}{*}{ No. } & \multicolumn{4}{|c|}{ Factor and level } & \multirow{2}{*}{$\begin{array}{c}\text { DAP } \\
\text { Yield/\% }\end{array}$} \\
\hline & $T /{ }^{\circ} \mathrm{C}$ & $t / \mathrm{h}$ & $r$ & $m$ & \\
\hline 1 & 1 & 1 & 1 & 1 & 54.9 \\
\hline 2 & 1 & 2 & 2 & 2 & 68.3 \\
\hline 3 & 1 & 3 & 3 & 3 & 69.8 \\
\hline 4 & 1 & 4 & 4 & 4 & 52.1 \\
\hline 5 & 2 & 1 & 2 & 3 & 75.5 \\
\hline 6 & 2 & 2 & 1 & 4 & 75.9 \\
\hline 7 & 2 & 3 & 4 & 1 & 68.4 \\
\hline 8 & 2 & 4 & 3 & 2 & 73.1 \\
\hline 9 & 3 & 1 & 3 & 4 & 77.2 \\
\hline 10 & 3 & 2 & 4 & 3 & 66.2 \\
\hline 11 & 3 & 3 & 1 & 2 & 78.9 \\
\hline 12 & 3 & 4 & 2 & 1 & 81.7 \\
\hline 13 & 4 & 1 & 4 & 2 & 53.1 \\
\hline 14 & 4 & 2 & 3 & 1 & 63.3 \\
\hline 15 & 4 & 3 & 2 & 4 & 68.1 \\
\hline 16 & 4 & 4 & 1 & 3 & 65.9 \\
\hline$H_{1}$ & 61.275 & 65.175 & 68.900 & 67.075 & \\
\hline $\mathrm{H}_{2}$ & 73.225 & 68.425 & 73.400 & 68.350 & \\
\hline $\mathrm{H}_{3}$ & 76.000 & 71.300 & 70.850 & 69.350 & \\
\hline$H_{4}$ & 62.600 & 68.200 & 59.950 & 68.325 & \\
\hline$R$ & 14.725 & 6.125 & 13.450 & 2.275 & \\
\hline
\end{tabular}


考察温度对反应收率的影响. 由表 2 可知, 4,7-二苯 基-1,10-菲啰啉的收率开始时是随着温度的升高而增加. 这是由于随着温度的升高, 反应速度加快, 导致收率也 逐渐增加; 但在温度超过 $120{ }^{\circ} \mathrm{C}$ 时, 收率又开始下降. 这可能是由于氧化剂 $\mathrm{I}_{2}$ 在高温下有所挥发所致.

考察时间对反应收率的影响. 由表 2 可知, 时间对 该反应也有一定的影响. 首先, 随着时间的增加, 4,7-二 苯基-1,10-菲啰啉的收率明显增加, 在 $2.5 \mathrm{~h}$ 处达到最高; 时间继续增加，收率反而有所下降.

考察 3-氯苯丙酮和 4-苯基-8-氨基喹啉进料的物质 的量比对反应收率的影响. 由表 2 可知, 3-氯苯丙酮与 4-苯基-8-氨基喹啉进料物质的量比为 $1.5: 1$ 为最佳, 3氯苯丙酮继续过量反而使得 4,7-二苯基-1,10-菲啰啉的 收率降低, 过量的越多, 产物收率降低的程度越大.

考察氧化剂用量对反应收率的影响. 由表 1 可知, 催化剂用量的增加有利于 4,7-二苯基-1,10-菲啰啉的生 成, 在 $8 \%$ 处达到最高. 从成本和收率出发, 选择催化剂 量为 $8 \%$.

基于表 2 和以上各种因素的讨论, 可以确定最优反 应条件为: 反应温度 $120{ }^{\circ} \mathrm{C}$, 反应时间 $2.5 \mathrm{~h}, \mathrm{I}_{2} / \mathrm{KI}$ 用量 为 $8 \%, 3$-氯苯丙酮与 4 -苯基- 8 -氨基喹啉进料的物质的 量比 $1.5: 1$. 在此条件下经多次重复实验得: 4,7-二苯
基-1,10-菲啰啉的平均收率为 $82.3 \%$.

\section{3 结论}

研究了以 4-苯基-8-硝基喹啉为起始原料, 经还原 得到 4-苯基- 8 -氨基喹啉(1), 通过 Skraup 反应合成了 4,7-二苯基-1,10-菲啰啉(2). 本实验所采用的氧化剂 对环境友好, 而且单步收率均较高.

通过正交实验，确定了反应的优化条件是：3-氯苯 丙酮与 4-苯基- 8 -氨基喹啉进料的物质的量比 $1.5: 1$, 催 化剂 $\mathrm{I}_{2} / \mathrm{KI}$ 用量为 $8 \%$ ，反应时间 $2.5 \mathrm{~h}$ ，反应温度 120 ${ }^{\circ} \mathrm{C}$. 由极差分析知道, 对反应收率影响因素由强到弱依 次为反应温度、3-氯苯丙酮与 4-苯基- 8 -氨基喹啉进料的 物质的量比、反应时间、催化剂用量. 在优化后的反应 条件下, 4,7-二苯基-1,10-菲啰啉的收率约为 $82.3 \%$.

\section{References}

[1] Sammes, P. G.; Yahioglu, G. Chem. Sov. Rev. 1994, 23, 327.

[2] Sahai, R.; Morgan, L.; Killema, D. P. Inorg. Chem. 1988, 27, 3495.

[3] Case, F. H. J. Org. Chem. 1951, 16, 1541.

[4] Case, F. H.; Strohm, P. F. J. Org. Chem. 1962, 27, 1641.

[5] Choo, D. C.; Seo, S. Y.; Kim, T. W.; Jin, Y. Y.; Seo, J. H.; Kim, Y. K. J. Nanosci. Nanotechnol. 2010, 10, 3614. 